\title{
Formulation of SPH for Discretizing the Fractional Differentiation
}

\author{
Hiroyuki Kisu, Guiming Rong and Yoshihiro Kondo \\ Graduate School of Engineering, Nagasaki University, Bunkyo-machi 1-14, Nagasaki 852-8521, Japan
}

\begin{abstract}
This study is a basic research for introducing the SPH as a solution to the fractional differential equation, because the SPH method has the flexibility in the boundary representation. To begin with, the discretized form for the integration is presented in addition to the conventional one for differentiation by SPH, because both the integration and differentiation are needed in the fractional differentiation. Then the formulation for the fractional differentiation by SPH is proposed by combining these two formula. The discretization formulation is applied to several concrete functions and the accuracy of their derivatives and primitive functions is examined numerically. These results are compared with those from FDM and FEM. It is found that the presented formulation has the sufficient accuracy to calculate the fractional differential equation.
\end{abstract}

Keywords: Smoothed particle hydrodynamics (SPH), Fractional differentiation, Diffusion problem, Finite Difference Method (FDM), Finite Element Method (FEM), Accuracy

\section{INTRODUCTION}

The fractional differential equation is suitable for describing problems which differ from the usual measure in space and the time, such as the diffusion problem in inhomogeneous mediums[1]. For a numerical solver of these problems, we try to introduce the SPH. As a basic research, this study aims at evaluating the possibility of SPH in numerically estimating the fractional differentiation.

The fractional differentiation requires the integral operation in addition to the differentiation operation. We also examine the accuracy of integration by SPH. Therefore, at first, the discretized form for the integration is presented in addition to the conventional one for differentiation by SPH. Then the formulation for discretization of the fractional differentiation by SPH is proposed by combining these two formula. The present formulations are applied to several concrete functions and the accuracy of their derivatives and primitive functions is examined numerically. The results are compared with those from the Finite Difference Method[2] and the Finite Element Method.

\section{FRACTIONAL DIFFERENTIATION}

The differential order $n$ in the $n$-th derivative, which is usually an integer, can be extended to a noninteger. The fractional differential equation is successfully applied to the diffusion problem in inhomogeneous mediums[1], for example. There are various representations for the fractional differentiation. In this study, we employ the definition of Riemann-Liouville [2] which is as follows:

$$
D_{x}^{p} f(x)=\frac{1}{\Gamma(p)}\left(\frac{d}{d x}\right)^{n_{p}} \int_{a}^{x}(x-y)^{n_{p}-p-1} f(y) d y .
$$

Here, $D_{x}^{p} f(x)$ is the $p$ th differential operator for a function $f(x), p$ is a noninteger figure, $\Gamma(p)$ is the Gamma function and $n_{p}=[p]+1$, where [ ] denotes the Gauss symbol. The lower limit for integration is set to zero in this study.

Equation (1) is given by the central difference form [2] as the following equation:

$$
D_{x}^{p} f(x)=\left(\frac{x-a}{M}\right)^{-p} \frac{1}{\Gamma(p)} \sum_{k=0}^{M-1} \frac{\Gamma(k-p)}{\Gamma(k+1)} f\left(x-\left(k-\frac{p}{2}\right) \frac{x-a}{M}\right),
$$

where $M$ is the dividing number of interval [a, $\mathrm{x}]$.

\footnotetext{
1 kisu@nagasaki-u.ac.jp
} 


\section{FORMULATION BY SPH FOR DIFFERENTIATION AND INTEGRATION}

In calculating the fractional derivatives, as found in Eq. (1), both of the difference operation and integral operation are required. The integration is also needed in the discretization formulation by SPH. This study intends to propose the integration by SPH and apply it to the calculation for fractional derivatives.

\section{Formulation for Differentiation}

The discretization formulation by SPH of derivatives has been proposed [3].The first spatial derivative as well as the second derivative can be expressed by the following equations, respectively, with the kernel function $W$ :

$$
<\nabla f(x)>=-\sum_{J=1}^{N} \frac{m^{J}}{\rho^{J}} f\left(x^{J}\right) \nabla W(z, h), \quad \quad<\nabla^{2} f(x)>=\sum_{J=1}^{N} \frac{m^{J}}{\rho^{J}} f\left(x^{J}\right) \nabla^{2} W(z, h) .
$$

Here, $m^{J}$ and $\rho^{J}$ is the mass and the density of particle $J(=1,2, \cdots, N)$ iijŇrespectively, $N$ is the number of particles in the influence area and $r$ is the distance between the two particles $I$ and $J$, and $<>$ indicates the kernel approximation operator. In this study, the kernel function with the following type is employed:

$$
W(z, h)=\left\{\begin{array}{ll}
\frac{2}{3 h}\left(1-\frac{3}{2} z^{2}+\frac{3}{4} z^{3}\right) & (0 \leq z<1) \\
\frac{1}{6 h}(2-z)^{3} & (1 \leq z<2) \\
0 & (z \geq 2)
\end{array}, \quad z=\frac{\mathbf{r}}{h}=\frac{\left|x^{I}-x^{J}\right|}{h},\right.
$$

where $h=\kappa r(\kappa \geq 1)$ is the smoothing radius defining the influence area of the kernel function $W$. The derivative of kernel function is expressed as

$$
\nabla W=\frac{x^{I}-x^{J}}{r} \frac{\partial W}{\partial r}
$$

\section{Formulation for Integration}

Integration must be performed in calculating the fractional differential equation defined in Eq. (1). Generally, the integral is not restricted to elementary functions. Similar to the formulation steps for differentiation, the integration for a function $f$ can be finally performed by integrating the kernel function:

$$
<F(x)>=\sum_{J=1}^{N} \frac{m^{J}}{\rho^{J}} f\left(x^{J}\right) \tilde{W}(z, h), \quad \tilde{W}(z, h)=\int W(z, h) d x,
$$

where $F(x)$ is the integrated form for $f$ and $\tilde{W}(z, h)$ is the integrated form of kernel function $W(z, h)$. Its concrete expression is omitted here for space limitation.

\section{Formulation for Fractional Differentiation}

Then the fractional differentiation can be expressed as the follows:

$$
\begin{aligned}
{ }_{a} D_{x}^{p} f(x) & =\left(\frac{d}{d x}\right)^{n_{p}}\left\{\frac{1}{\Gamma\left(n_{p}-p\right)} \int_{a}^{x}(x-y)^{n_{p}-p-1} f(y) d y\right\}=\left(\frac{d}{d x}\right)^{n_{p}}\left\{\frac{1}{\Gamma\left(n_{p}-p\right)} \sum_{J=1}^{N} \frac{m^{J}}{\rho^{J}} G\left(x^{J}\right) \tilde{W}(z, h)\right\} \\
& =-\sum_{J=1}^{N} \frac{m^{J}}{\rho^{J}} V\left(x^{J}\right) \nabla W(z, h),
\end{aligned}
$$

where

$$
V(x)=\frac{1}{\Gamma\left(n_{p}-p\right)} \sum_{J=1}^{N} \frac{m^{J}}{\rho^{J}} G\left(x^{J}\right) \tilde{W}(z, h), \quad G(x)=\int(x-y)^{n_{p}-p-1} f(y) d y .
$$




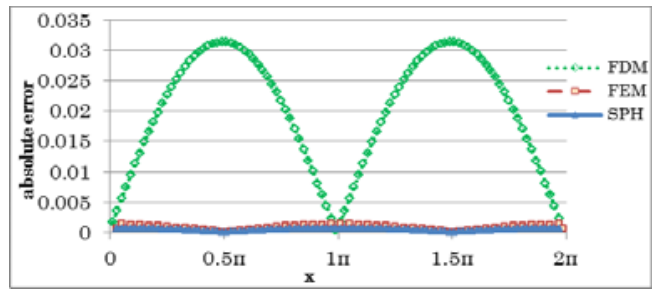

FIGURE 1. Absolute error of each method in calculating derivative of $\sin (\mathrm{x})$

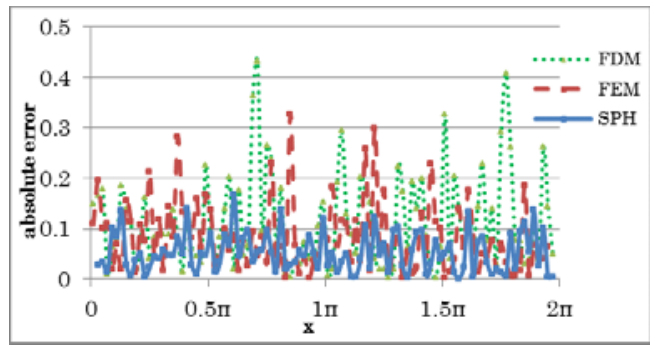

FIGURE 2. Absolute error of each method in calculating derivative of $\sin (\mathrm{x})$, when errors included in primitive function

\section{INVESTIGATION OF PERFORMANCE OF SPH}

In this section, $h=1 r$ is used for calculation, and the domain is discretized evenly over 100 nodes. The precision of SPH may be influenced by dividing number, the ratio of $h$ to $r$, and others. These will be discussed in other paper.

\section{Performance of SPH for the usual differentiation}

Several examples were tested in order to verify the performance of SPH in the accuracy for the usual differentiation. We show here one case that the test function is $f(x)=\sin x$. That is, numerical results from FDM, FEM and SPH are compared with the exact derivative function, $\cos x$, being with the same dividing number. The results are shown in figure 1. It can be seen that under present conditions, the results from SPH are better than those from FDM, while are almost the same, or better than those from FEM.

Next, supposing the case that the function values at the dividing points, $f\left(x_{i}\right),(i=$ a dividing point number $)$, may contain some errors, we investigate the behavior of the numerical results. The results are displayed in figure 2 . We can see that the accuracy from SPH keeps the best.

The cases of $f(x)=e^{x}$ and $f(x)=\log (x+1)$ were also tested and the behavior of accuracy is similar.

\section{Performance of SPH for the usual integration}

For the test of integration, the same function, $f(x)=\sin x$, is adopted. The results are shown in figure 3, being compared with the right function, $-\cos x$. It is found that the accuracy is good. We also calculated it by FEM and the tendency remains unchanged.

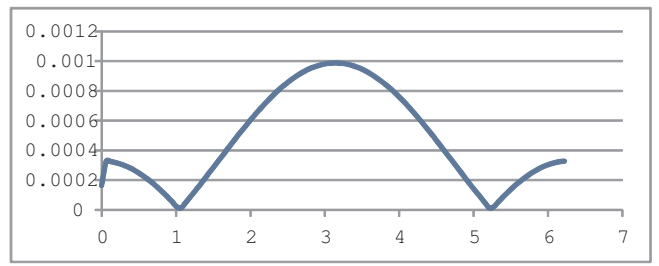

FIGURE 3. Absolute error in calculating $\int \sin x d x=-\cos x+1$ by SPH 


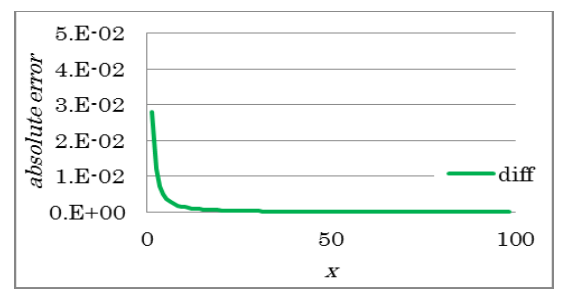

FIGURE 4. Absolute error in calculating 0.5th differentiation by SPH

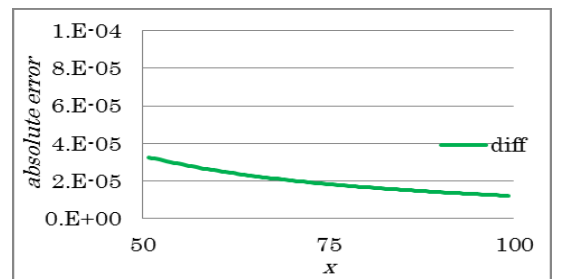

FIGURE 5. Absolute error in calculating 0.5 th differentiation by SPH, in case of $x \geq 50$

\section{Performance of SPH for the fractional integration}

For confirming the case of fractional differentiation, we set test function as $f(x)=x$ and calculate for its 0.5 th differentiation. It is said that the central difference method will give good accuracy for this problem, therefore we take this method for comparison. The analytical solution in this case is expressed as the follows:

$$
D_{x}^{0.5} f(x)=2 \sqrt{\frac{x}{\pi}}
$$

We calculated this example by two methods, FDM[2] and SPH. The results from SPH are shown in Figure 4, being compared with equation 9. It may be noticed that the accuracy becomes suddenly lower in the vicinity of $x=0$. The considered reason for this phenomenon is that the differential coefficient of the exact solution of equation 9, as easily seen, will be singular at $x=0$. In fact, in the interval which excluded such a "special" point, the calculated values by $\mathrm{SPH}$ have good precision as shown in figure 5, which is almost the same with the FDM.

\section{CONCLUSIONS}

This paper presents a basic research for establishing the SPH as a tool for solving fractional differential equation, from the viewpoint of making the SPH become a general-purpose numerical analysis method. We investigated the basic performance of SPH in the calculation of both differentiation and integration, because these operations are all needed in the evaluation of fractional differentiation. In addition to the general operation of discretization for differentiation in SPH, we proposed a new discretization formulation for integration. The discretization formulation by SPH for the fractional differentiation is proposed based on the two operations. It is confirmed by the numerical calculation that the accuracy of presented method is sufficient for calculating the fractional differential equation.

\section{REFERENCES}

1. N. Shimamoto, âĂIJModeling by the fractional differential equation for diffusion phenomena in inhomogeneous mediums, simulationâĂİ, Mathematical seminar, vol.12, pp.60-64, 2011.

2. T. Okayama, S. Murashige, âĂIJNumerical calculation of fractional derivatives using automatic differentiation and double exponential formulaâĂIiij ŇBulletin of the Japan Society for industrial and applied mathematics, Vol.16, No.1 (2006), pp.51-65

3. G. R. Liu, M. B. Liu, Smoothed Particle Hydrodynamics-A mesh free particle method, World Scientific, 2003. 